\title{
Zu den Werken des Peter von Eboli. Von
}

\author{
Robert Ries.
}

I.

In der Einleitung zu seiner Ausgabe des "Liber ad honorem Augusti“ des Peter von Eboli1) wirft Siragusa auch die schon mehrfach $^{2}$ ) erörterte Frage wieder auf, welches Thema das uns nur durch eine kurze Äusserung des Dichters selbst bekannte zweite Werk Peters behandelt habe; er kommt nach eingehender Prüfung (Prefazione, p. XX-XXIII) zu dem Ergebnis, dass wahrscheinlich (er entscheidet sich nicht unbedingt; cf. XXIII) in diesem Werk die Taten Friedrichs II. besungen worden seien.

1) Liber ad honorem Augusti di Pietro da Eboli secondo il cod. 120 della Biblioteca Civica di Berna a cura di G. B. Siragusa. (Tavole Roma 1ᄃ05), Testo Roma 1906 = Istituto Storico Italiano, Fonti per la storia d'Italia, vol. 39, (40).

2) Es seien hier gleich im Zusammenhang die Titel der wichtigsten einschlägigen Bücher, bezw. Artikel, die in unserer Untersuchung mehrfach zitiert werden, zusammengestellt:

A. Huillard-Bréholles, Notice sur le véritable anteur du poëme, De Balneis puteolanis et sur une traduction française inédite du même poëme, lue à la séance du 19. décembre 1851 in: Mémoires de la Société Nationale des Antiquaires de France, Tome XXI (Troisième Série, Tome premier), Paris 1852, p. 334-353.

Salvatore de Renzi, Collectio Salernitana, 'l'omo primo (Napoli 1852), p. 280 -291, Tomo secondo (Napoli 1853), p. 780-782.

Henry Simonsfeld, Venetianische Studien: I: Das Chronicon Altinate, München 1878 , p. $70-74$. 
Auf Grund des uns vorliegenden überaus dürftigen Materials durch einen lückenlosen Beweis hier endgiltig das letzte Wort zu sprechen, dürfte nach meiner Meinung allerdings kaum möglich sein; jedenfalls aber scheint mir Siragusas Lösungsversuch nicht das Richtige zu treffen, so dass ich ein nocbmaliges Prüfen der Frage für lohnend halten möchte.

Leider steht uns für unsere Untersuchung im Grunde nur eine einzige Quelle zur Verfügung: tine Stelle in dem Gedicht ,De Balneis (Puteolanis)“ 1), einem Sang über die Bäder von Pozzuoli und der Terra di Lavoro, in dem die Namen und heilungbringenden Eigenschaften der einzelnen Bäder in 35 (mit Einleitungs- und Schlussstrophe 37)

Block $I=$ Paul Block, Petrus de Ebulo und seine Nachrichten über die Gemahlin Kaiser Heinrichs VI. Programm des Gymnasiums und Realgymnasiums zu Prenzlau, Prenzlau, 1883.

Block II = Paul Block, Zur Kritik des Petrus de Ebulo, II. Teil, Greifswalder philos. Inaug.-Diss., Prenzlau, 1883.

Erasmo Percopo, I bagni di Pozzuoli, poemetto napolitano del secolo XIV, Archivio storico per le province Napoletane XI (Napoli 1886) p. 597-750 (bezw. 635), bes. p. 609-623.

Guido Bigoni, Una fonte per la storia del regno di Sicilia: Il Carmen di Pietro da Eboli, Genova 1901, bes. p. 9-14.

Rota $=$ Petri Ansolini de Ebulo De rebus Siculis Carmen a cura di Ettore Rota (= Rerum Italicarum Scriptores. Raccolta degli storici Italiani ... ordinata da L. A. Muratori. Nuova edizione riveduta, ampliata e corretta con la direzione di Giosue Carducci e Vittorio Fiorini. Tom. XXXI, P. I) Città di Castello, 1904 (Druckabschluss 1909), Prefazione, bes. p. XIX-XXVIII.

F. Marletta, Pietro da Eboli è Pietro Ansolino? - Archivio storico per la Sicilia orientale, Anno III (Catania 1906), p. 107-117.

Es sei mir erlaubt, auch an dieser Stelle meinem hochverehrten Lehrer Herrn Professor Dr. Karl Hanpe für seine mir freundlicbst erteilten Ratschläge meinen verbindlichsten Dank zu sagen.

1) Für den ersten (unvollständigen) Druck in der Ausgabe des im XIII. Jahrhundert verfassten und von Francesco Aretino wieder aufgefundenen, Libellus de mirabilibus civitatis Putheolorum et locorum vicinorum ac de nominibus virtutibusque balneorum ibidem existentium" - recollectum et Impressum per Arnaldum de Bruxella in Civitate Neapolis. . . Die nitimo mensis Decembris Anno a nativitate domini MCCCCLXXV^, einen seltenen mir nicht zugänglichen Inkunabelndruck, verweise ich auf Percopo 610 Anm. 2 u. 618 (und Anm. 2) -- (nach Percopo 619 ist dieser erste Druck übrigens nicht von dem neapolitanischen Arzt Joh. Elisius herausgegeben, wie noch Huillard-Bréholles 335 annahm; Elisius gab nur einen speziell auf die Bäder bezüglichen Auszug daraus; cf. Percopo 619 Anm. 1). Ich verzichte auch hier darauf, im Einzelnen auf die gesamte Druckgeschichte des Gedichts „De Balneis e einzugehen: vgl, darüber: Paulli M. Paciaudi C. R. Historici Ordinis Hierosolymitani Regiaeq. Parisieneis Academ. In- 
Epigrammen (zu je 12 Versen) beschrieben werden 1 ) und dessen Verfasser, wie besonders Block ${ }^{2}$ ), vor allem durch stilkritische Untersuchung, schlagend nachgewiesen hat, eben Peter von Eboli ist. Die Stelle lautet wörtlich in dem zuletzt von Siragusa ${ }^{3}$ ) nach den besten Lesarten hergestellten Text ${ }^{4}$ ):

1. Suscipe, Sol mundi, tibi quem presento libellum;

De tribus ad dominum tertius iste venit.

Primus habet patrios civili marte triumphos.

Mira Federici gesta secundus habet.

5. Tam loca quam vires quam nomina pene sepulta

Tertius Euboycis iste reformat aquis.

Cesaris ad laudem tres scripsimus ecce libellos:

Firmius est verbum quod stat in ore trium.

Si placet, annales veterum lege, Cesar, avorum;

10. Pauper in Augusto nemo poeta fuit.

Ebolei vatis, Cesar, reminiscere vestri,

Ut possit nati scribere facta tui.

Es fragt sich zunächst, an wen sich der Dichter mit dieser Wid. mung wendet. Da V. 3 zweifellos auf Peters bekanntestes Werk: den

script. et humanar. literar. sodalis De sacris Christianorum balneis liber singularis secundis curis emendatior et auctior. Romae MDCCLVIII, p. 50, Huillard-Br. 335 und die ausfübrlichen und erschöpfenden Ontersuchungen von Percopo, bes. p. 610 Anm. 2 und 618-620, z. T. wiederholt von Rota XXVI, der nichts Neues hinzufügt. Die mehr oder weni ģer freien Übertragungen des Gedichts im XIV. Jahrhundert in die neapolitanische Vulgär- und in die französische Sprache (vgl. die Zusammenfassung bei Percopo 620) sind im Rahmen dieser Untersuchung nicht zu berẗckeichtigen. - Für das ganze Gedicht, vorläufig (cf. Marletta 107 Anm. 3) am besten zu benutzen: die Ausgabe von Capaccio $I=$ Balnerarum, quae Neapoli, Puteolis, Baiis, Pithecusis extant, virtutes .... Neapoli MDCIV [Anhang zu Puteolana Historia a Julio Caesare Capacio; Neapoli MDCHII mit selbständiger Paginierung, $4^{\circ}$ ], p. 69-84, wiederabgedruckt: Capaccio $\mathrm{II}=$ La vera antichità di Pozzuolo descritta da Giulio Cesare Capaccio . . . . in Napoli MDCVII, 80, 328348. Vgl. auch Abschnitt II.

1) cf. V. 5 der sogleich wiederzugebenden Strophe.

2) Block I, 11-14. Allerdings hat schon Capaccio anf Grund der ihm vorliegenden Handschrift auf die Autorschaft eines Ebolitaners hingedeutet: I, 4, ef. II, 327; und Huillard-Br. 339, 344 und De Renzi I, $286 \mathrm{ff}$. u. II, 780 f. hatten diesen Sachverbalt bereits ganz erkannt und dargelegt vor Block, der dann den ausführlichen Beweis erbrachte.

s) pref. XIX f. (Es ist die Schlussstrophe des ganzen Gedichts, nach Cod. XIII, C, 37 der Nationalbibliothek Neapel, c. 49:, conclusio auctoris`; ct. Siragusa XIX, Anm. 6.) Derselbe Text wurde schon vorher in wörtlicher Übereinstimmung von Huillard-Br. $338 \mathrm{f}$. gebracht und von Rota $\mathrm{XXV}$ wiederholt (von diesem unter wohl versehentlicher Auslassung von V. 9 u. 10 und in V. 3 mit der Veränderung ,Primus habet partos.... - darüber handelt Abschnitt II, Teil II ausführlicher.)

4) Siehe Abschnitt II, Teil I. 
"Liber ad honorem Augusti " hindeutet, der ja Heinrichs VI. Eroberung Siziliens besingt, ferner die Fassung dieses Verses, wie ich durch Zusammenstellung der mir bekannten Lesarten ${ }^{1}$ ) bewiesen $z u$ haben hoffe, gesichert erscheint, ergibt sich als notwendige Folgerung, dass wir als den in unseren Versen Angeredeten Friedrich II. anzusehen haben ${ }^{2}$ ). Diese Folgerung zieht denn auch Siragusa (XII). Im Gegensatz zu ihm sieht Block ${ }^{3}$ ) auf Grund der auf den ersten Blick sehr verlockenden Fassung: "partos civili Marte triumphos" die Widmung als an Heinrich VI. gerichtet an und kann infolgedessen garnicht umhin (ein richtiges Ergebnis auf falschem Wege!), die Gesta Federici (weil v or dem Gedicht „De Balneis" geschrieben) für Friedrich Barbarossa in Auspruch zu nehmen. A ber diese Fassung ist, wie gesagt, durch keine uns bekannte Handschrift oder Lesart belegt uud muss deshalb fallen gelassen werden; sie ist auch deshalb zu verwerfen, weil dann jede Andeutung fehlen würde, um wessen Triumphe es sich handelt, und wir dann tuos (künstlich) zu ergänzen hätten. Trotz dieses Tathestandes nimmt Rota4) die Vermutung Blocks wieder auf, ohne auf die handschriftliche Überlieferung des Verses näher einzugehen, indem er erklärt: „Il „patrios triumphos* di qualche edizione non infirma quanto

1) Siehe Abschnitt II, Teil II.

2) Das tat schon Capaccio I, 4 auf Grund des ihm vorliegenden Manuskripts (vgl. Anmerkung 4); cf. Capaccio II, 327; vgl. ferner G. Tiraboschi, Storia della letteratura Italiana, Tomo quarto (Modena 1774), Libro III, $3 \check{2} \mathrm{f}$., Percopo 613f. - Es ist auch schon darauf hingewiesen worden, dass Friedrich II. später tatsächlich die Bäder von Pozzuoli aufgesucht hat (Anfang Oktober 1227): Rycc. de S. Germano, MG. SS. XIX, 348: ... Imperator de Apulia tune venit ad balnea Puteoli...; cf. G. Paolucci, La prima lotta di Federico II di Svevia col papato (Atti della Reale Academia di scienze, lettere e belle arti di Palermo. Terza Serie (Anni 1902-1903), Vol. VII, Palermo 1904), p. 18 u. Anm. 1.

3) Block II, 2-4. Für seine paläographisch naheliegende Emendation kann er freilich keinen Beleg in der Überlieferung nambaft macben. Durch ihn beeinflusst war wohl auch K. A. Kehr; er schreibt in der Besprechung von Bigonis Buch (cf. S. 576 Anm. 2): Deutsche Literaturzeitung, XXIII. Jahrgang (Leipzig 1902) col. 673 : ,Die Schluss- und Widmungsstrophe des Gedichts .. bezieht Bigoni auf Friedrich II. Aber spricht nicht einiges vielmehr für Heinrich VI.? -

4) Rota XXVII. Einen neuen Beweis allerdings sucht er für seine Auffassung geltend zu machen : „Infatti nell' edizione veneta, sopra citata (d. h. Venetiis apud Juntas, 1553; ef. ebd. XXVI - dieser Druck war mir nicht zugänglich, vielmehr nur aus Percopo 610 Anm. 2 (611) bekannt) il carme è intitolato, ad Henricum imperatorem *, e nessuna importanza si deve attribuire a ciò che un secolo dopo (! - nämlich 1604) scriveva il Capaccio: ... Ea, quae circumferuntur (nämlich unser Gedicht) .... scripta sunt .... ab Ebolitano quodam qui Federico Regi opus illud dicaverat, id quod apud me testatur manuscriptus is. dex ..." (das Zitat ron mir vervollständigt nach Capaccio I, 4), dato falso che 
dicemmo, perchè alcuni codici dànno „partes" (ediz. Capaccio), evidente corruzione di "partos", come il De Renzi ${ }^{1}$ ) ben vorrebbe correggere. Il -patrios" fu un enendazione(!) di chi rolle(!) che il poemetto fosse dedicato a Federico II ...." - die Folge ist, dass er (XXVIII) zu dem sehr bestimmten Ergebnis kommt: "Il „De Balneis" fu presentato nel 1197 ad Eurico VI coll' augurio di illustrare in un quarto Poema le glorie di Federico II." Trotz dieser Behauptung Rotas müssen wir aus den angeführten Gründen mit Siragusa daran festhalten, dass das Gedicht „De Balneis“ Friedrich II. gewidmet ist. Damit scheint mir im Grunde aber auch schon erwiesen zu sein, dass jenes zweite nicht auf uns gekommene Gedicht Peters, die "Gesta Federici", von denen V. 4 redet, nicht ebenfalls auf Friedrich II. bezogen werden, nicht seine Taten besungen haben kann: denn da er noch drei Verse vorher mit "tibi" angeredet wird, würde es doch jedem natürlichen Sprachgebrauch widersprechen, wenn eben er jetzt hier in der 3. Person eingeführt werden würde 2 ). - Wenn Siragusa diesen Einwand von vornherein zu entkräften sucht (XXf.), indem er darauf hinweist, dass Peter ja auch innerhalb eines Satzes nebeneinanderstelle:

(V. 1 u. 7) „tibi presen to... ${ }^{\star}-$, tres scripsim u s ecce libellos ${ }^{\star}$, oder (V. 11 u. 12) „Ebolei vatis, Cesar, reminiscere vestri,

ut possit nati scribere facta $t u$ i « (wobei ich übrigens das. "vestri ${ }^{*}$ auf Friedrich II. und Heinrich VI. beziehe und dadurch den Plural durchaus bedingt finde) - so scheint mir diese Feststellung alles eher als schlagend: Abwechslung zwischen derselben Person Singularis und Pluralis und Abwechslung zwischen der zweiten und dritten Person stehen denn doch nicht auf derselben Stufe.

Es sind aber noch mehr der Gründe, die gegen Siragusas Hypothese sprechen: der Dichter setzt in den angeführten Versen zweifellos die "Gesta Federici* als Friedrich II. von früher her bekannt voraus: was für „Taten Friedrichs II.“ könnten das überhaupt sein? -

probabilmente si intrecciò col fatto che Federico II nel 1227 si servì dei bagni di Pozzuoli per ristabilirsi in salute - diese Feststellung kann unsere Au fassung natürlich nicht erschüttern, da der um 50 Jahre spätere Druck Capaccios eben auch auf eine selbständige alte Handschrift zurückgeht (deren Alter wir allerdings ebensowenig kennen wie das der Handschrift für den 1553-Druck), mithin beide Drucke für uns gleich en Quellenwert besitzen.

1) Das trifft übrigens nicht zu und muss auf einem lrrtum beruhen: De Renzi gibt (I, p. 289 u. 290) die Fassung von Huillard-Bréholles: „patrios " wieder.

2) Vgl, auch schon Huillard-Br. 340; De Renzi 1, 290; Simonsfeld 71, Anm.3; Percopo 614; gegen eine Beziehung auf Friedrich II. siehe ferner die Andeutungen Marlettas, Arch. Stor. per la Sic. orient., Anno VI (Catania 1909), $419 \mathrm{f}$. 
Da Peter ron Eboli, wie es seine Dichtungen beweisen und wie es Siragusa selbst zugibt (XXII), uns entgegentritt als „un uomo, che viveva nell'Italia meridionale e il cui mondo era limitato a quelle regioni, delle quali soltanto conosceva i luoghi, gli uomini, gli usi, le vicende...", so würden sich doch etwa von ihm beschriebene "Gesta“ Friedrichs II. auch nur mit Taten des jungen Fürsten beschäftigt habeu können, die sich im sizilischen Königreich abspielten: aber diese "Taten" Friedrichs II. in Sizilien beginnen eigentlich erst mit dem Ende des Jahres 12201), als er aus Deutschland in den Süden zurückkehrte (die Wirren während Friedricus Jugendzeit bis 1212 konnte selbst der ruhmreduerischste Dichter nicht "mira gesta " nennen²), also ganz kurz vor dem Zeitpunkt, wo uns unser Dichter schon als verstorben bezeugt ist. (Februar 1221 in der Urkunde Friedrichs II. für das Erzbistum Salerno; BF. 1280 ${ }^{3}$ ).

Dass wir es also bei dem uns leider nicht überlieferten zweiten Werk des Peter von Eboli mit ,Gesta" Friedrichs II. zu tun hätten, ist nach alledem so gut wie ausgeschlossen; dagegen dürften wir (und hier möchte ich eine Vermutung Blocks ${ }^{4}$ ) wieder aufnehmen) in den verlorenen "Gesta" einen Sang über Friedrich Barbarossas Kreuzzug zu sehen haben; zu dieser Annahme passt auch gut die Bezeichnung: „mir a gesta“, - die wunderbaren Taten Barbarossas auf seinem Kreuzzug, und Siragusas Einwand: „che il mondo di Pietro da Eboli era ristretto all'Italia del Mezzogiorno" (XXI; cf. XXIII), verliert so stark an Schlagkraft; denn bei der Behandlung dieses Themas kam es nicht so sehr auf genaue Orts- und Sachkenntnis an (jedenfalls bedeutend weniger als bei der von Siragusa ${ }^{5}$ ) angenommenen Schilderung der

1) 1220 Dezember 13. Ankunft in S. Germano. Böhmer-Ficker, Regesta imperii V, I, 1258 a (hinfort BF. abgekürzt.). Noch in Dezember in Suessa (BF. 1260a), darauf in Capua (Hoftag BF. 1260 b) und Neapel (bis 1221 Januar 4; BF. $1261 \mathrm{ff}$.).

2) Vgl. schon Huillard-Br. 340:, l' expression, mira gesta serait d'une exagération trop évidente , ihm folgend Simonsfeld, 71 Anm. 3 (72). - Der Ausdruck, mira gesta deutet doch wohl auch im Gegensatz zu den, civili marte triumphos a auf eine Unternehmung der auswärtigen Politik, und endlich - wenn Peter wirklich Friedrichs II. Taten besungen hätte, würde er dann später die Widmung des Gedichts ,De Balneis " an denselben Herrscher in so allgemeinen Ausdrücken gehalten haben?

9) Siehe Abschnitt III.

4) Block I, 14f. und II, 3. Übernommen von Bigoni 13 und Rota XXVI.

5) XXIII. Nebenbei sei bemerkt, dass diese Taten Friedrichs II.: „la sua condotta a Genova e a Milano (?) e sopratutto l'audace entrata in Costanza ... , die Siragusa versehentlich zu 1210 ansetzt, dem Jahre 1212 angehören; cf. Winkel- 
Taten Friedrichs Ií. in Oberitalien und Deutschland (Constanz)), vielmehr konnte Peter hier eher seiner Phantasie und seiner Vorliebe für historisch Reminiszenzen $^{1}$ ) freien Lauf lassen; vor allen Dingen aber fällt bei dieser Annahme auch das ohnedies vicht allzu beweiskräftige ${ }^{2}$ ). Bedenken in sich zusammen, das Siragusa (XXI) als entscheidend hinstellt: "nè so come arrebbe potuto lodare le imprese (del Barbarossa) senza infierire, come è suo costume, contro i nemici di lui, fra i quali principalissimo doveva apparire a un citadino del regno di Sicilia quel re Guglielmo II che per Pietro da Eboli fu l'ideale della bontà, dellia bellezza, della virtù ..." - zur Polemik (ausser etwa gegen die Ungläubigen) war bei diesem Stoff gar keine Gelegenheit! -

Zum Belege für meine Auffassung könnte ich ferner auch mit. Block (I, 15) noch darauf hinweisen, dass wir bei Peter von Eboli zur Bearbeitung dieses Stoffes schon gewisse Ansätze im „Liber ad honorem Augusti " finden; vgl. V. 314-327 (Ausgabe Siragusas p. 29), V. 1582-1605 (ebd. p. 109 ff.); es kommt hinzu, dass Kaiser Friedrich Barbarossa wie im Gedicht "De Balneis" „Federicus" so auch hier ohne jedes Epitheton einfach „Fredericus“ genannt wird ${ }^{3}$ ).

Dagegen kann der Satz:

(V. 9) Si placet, annales veterum lege, Cesar, avorum ....,

wie mich im Gegensatz zu Huillard-Bréholles u. Block ${ }^{4}$ ) dünkt, ebensowenig für als gegen unsere These beweisen, er hat m. E. nur den allgemeinen Sinn: wie du aus der Geschichte deiner Vorfahren auf

mann, Jahrbücher Philipps von Schwaben und Ottos IV. von Braunschweig, Bd. II (1878), p. $320 \mathrm{ff}$.

1) Vgl. etwa V. 313 ff. (Ausg. Siragusas p. 29), die Peter leicht breiter ausmalen konnte.

2) Für die Stellung, die Peter der Persönlichkeit Friedrich Barbarossas gegenüber unbeschadet seiner Verehrung für die normannische Dynastie einnahm, ist doch bezeichnend V. 1378 (Ausg. Siragusas p. 95).

„Ex hinc Rogerius, hinc Federicus eris ‘....

in dem er den Namen des Grossvaters väterlicherseits: Friedrich und den des Grossvaters von mütterlicher Seite: Roger, die der junge Sohn Heinrichs und Constanzes tragen sollte, gleichwertig nebeneinander stellt. Vgl. auch Anm. 3.

3) V. 314 (Ausg. Siragubas p. 29), V. 1582, 1589, dazu 1580 (ebd. p. 109; cf. V. 1378, ebd. p. 95). Dazu vgl. die bildliche Darstellung Friedrich Barbarossas (Siragusas Ausgabe, 'lavole, Tav. XLIX, mittleres und unteres Bild, wo sich allerdings die Legende : Fredericus, Imperator e findet). - Nebenbei möchte ich bemerken, dass sicb auch in zwei Handschritten (vgl. Abschnitt II, Teil II) des Gedichts „De Balneis" die Form ,Fredericus" nachweisen lässt: Percopo 685 Anm. und Tiraboschi, Storia della letteratura Italiana, Tomo IV, Libro III, 352.

4) Huillard-Br. 341; Block I, 14. 
dem kaiserlichen Throne (wobei die römischen Kaiser ebenso wie die Kaiser deutscher Nation von Karl dem Grossen an nach des Dichters Auffassung ${ }^{1}$ ) mit unter diesen Begriff fallen dürften) sehen kannst:

(V. 10) Pauper in Augasto nemo poeta fuit.

(etwa zu übersetzen: „war vor dem Throne des besungenen Kaisers noch kein Dichter arm.")

Endlich möchte ich noch ein Bedenken erwähnen (um es zu zerstreuen), das Block ${ }^{2}$ ) geltend macht: aus V. 2 u. 7 gehe deutlich hervor, dass alle 3 Schriften an die gleiche Adresse gerichtet waren. Hier muss ich Siragusa ${ }^{3}$ ) durchaus beistimmen, dass in diesem Zusammenhang mit ,dominus" (V. 2) u. "Cesar" (V. 7) der Kaiser im Allgemeinen, man könnte sagen: der Kaiser als Begriff ("l'inperatore in genere ") gemeint sei (d. h. also, spezieller gefasst, alle 3 Kaiser). Allerdings muss uns die Tatsache, dass Friedrich II. dem Gesamtbegriff "Cesar", mit untergeordnet ist, ja dass er sogar direkt4) als "Cesar", Kaiser angeredet wird, zu denken geben (das ist meines Wissens bisher noch nicht beachtet worden): sie würde zunächst folgende Hypothese ausserordentlich nahe legen, die auch Blocks ${ }^{5}$ ) Befremden, dass wir von keiner Belohnung des Dichters durch Friedrich II. wissen, aufs Einfachste erklären würde.

Auf die Nachricht hin, dass Friedrich II. ins sizilische Königreich zurückkehren werde ${ }^{6}$ ), hält es Peter von Eboli für angebracht, um sich dessen Gunst zu erwerben, diesem das Gedicht "De Balneis* zu widmen. Die Bezeichnung „Caesar" wählt Peter in der richtigen Voraussetzung, dass Friedrich vor seixer Ankunft im Königreich die Kaiserkrone erlangt habon werde. Vielleicht ehe er Friedrich sein Gedicht überreicht hat, jedenfalls aber ehe dieser dem Dichter die erhoffte Belohnung zu Teil werden lassen kann, stirbt Peter von Eboli und ver-

1) Dass Peter in Caesar und Karl dem Grossen Vorfahren der staufischen Kaiser sah, beweist z. B. V. $312 \mathrm{ff}$. (Ausg. Siragusas p. 29) u. a. Anm. 2.

2) Block .II, 2; vgl. schon I, 14 Anm. 1 (15). Ihm folgend Rota XXVII u.

s) XXI Anm. 2 und XXII.

4) V. 9 uuserer Schluss- und Dedıkationsstrophe; ausserdem die beiden letzten Verse der Eingangsstrophe (die sonst keinerlei Bemerkung über den Aciressaten enthält):

,Quarum (sc. aquarum) virtutes et nomina, maxime Caesar,

presens pro vestra laude libellus habet ${ }^{\star}-($ Capaccio, l. c. I, $70==$ II, $328=$ Block I, 12 Anm. 1).

5) Block I, 14 Anm. 1 (15); vgl. II, 2.

6) Vgl, meine Anmerkung 1 Seite 581. 
macht in seinem Testament ${ }^{1}$ ) der Kirche ron Salerno das ihm einst von Heiurich VI. geschenkte Mühlengrundstück (molendinum de Albiscenda).

Aber diese zunächst verlockende These wird dadurch hinfällig; dass wir schon eine bisher für unsere Frage noch nicht genügend verwertete ${ }^{2}$ ) Urkunde Friedrichs von spätestens Anfang Juli 1220 für das Erzbistum Salerno besitzen (erwähnt in Urkunde Friedrichs II. für die Lente von Eboli v. 1220 Juli $3 \mathrm{Ulm}-\mathrm{BF} .1141)^{3}$ ), in der bereits das "molendinum Albescendae" bestätigt wird, und dass dadurch der Termin für Peters Tod bis mindestens in den Juni 1220 zurückversetzt wird. Aus dieser Tatsache ergibt sich, dass wir als zeitlicke Grenzen für die Vollendung und Überreichung4) des Gedichts „De Balneis" die Jahre 12115) u. 1220 (Juni) festzuhalten haben. Darüber hinaus eine genauere Datierung mit unbedingter Sicherheit zu geben ist unmöglich.

Immerhin möchte ich hier einer (allerdings nur hypothetischen) Ansicht Ausdruck geben, die mir einer gewissen Wahrscheinlichkeit nicht zu entbehren scheint.

1) Siehe Abschnitt III.

2) Für Siragusa. IX, Anm. 1, hat die Urkunde naturgemäss nur nebensächliche Bedeutung, da er ja fälschlich in BF. 1280 eine zeitlich frühere (weil im Februar 1220 ausgestellte) Urkunde sieht (vgl. Abschnitt III), und Block erwähnt sie (I, $34 \mathrm{Anm}$. l) nur beiläufig (ausserhalb seiner biographischen Zusammenfassung). In den übrigen Arbeiten ist die Urkiunde meines Wissens überhaupt nicht für unsere Frage herangezogen worden.

3) Gedruckt: Huillard-Bréholles, Hist. diplom. Frid. sec., tom. I, pars II, p. $797 \mathrm{f}$. (nach authentischer von den Richtern Lucas und Johannes ausgestellter Kopie der Urkunde für die Leute von Eboli von 1220 Oktober aus dem Register des erzbischöflichen Archivs zu Salerno): cf. dazu Siragusa IX, Anm. 1, der unseren Druck nicht zu kennen scheint. Die betreffende Stelle heisst (Huillard.Br. 1. c. p. 797) wörtlich: ... Noverit universitas vestra, quod ... concessimus et confirmavimus... molendinum Albescende cum aqueductu suo necnon omnia iura et rationes que Salernitane ecclesie in Ebulo et pertinentiis eius pertinent ..... - dies ist, so viel ich sehe, die erste Urkunde, in der die Mühle unter den Besitzungen des Erzbistums erscheint: die Bestätigungsurkunde Constanzes II. und Heinrichs (VII.) von 1216 Juni Messina (BF. 3844; gedruckt: Winkelmann, Acta imperii, I, $376 \mathrm{f}$., $\mathrm{n}^{0} 443$ ) zählt die Besitzungen des Erzbistums nicht einzeln auf, so dass wir daraus, dass die Mühle hier nicht genannt wird, nicht etwa unbedingt schliessen können, dass damals unser Dichter noch gelebt babe.

4) Das , presento c des V. 1 macht es immerhin wahrscheinlich, dass Peter sein Gedicht Friedrich II. selbst überreicht hat.

5) Der terminus post quem für die Ansetzung des Gedichts ist dadurch gegeben, dass der, natus", von dem V. 12 redet, d. h. Heinrich (VII.) erst 1211 geboren wurde (BF 3835 b); mir scheint es undenkbar, dass sich dieser Vers ant einen noch nicht geborenen Sohn beziehen könnte, was Siragusa XVIII für nicht unmöglich erklärt. 
Nachdem Friedrich von den deutschen Fürsten in Nürnberg im September 1211 mit Zustimmung des Papstes zum deutschen Kaiser gewählt worden war' $^{1}$ ) und die Wahl angenommen hatte 2 ), brachte er vor seinem Aufbruch nach Deutschland einen Monat (Mitte MärzApril) 1212 in Gaeta $\mathrm{zu}^{3}$ ). Allerdings war seine Lage auf dem sizilischen Festland in Augenblick keine glänzende, aber der Abzug Ottos IV. und die Unterstützung durch den Papst deuteten auf eine den Staufern günstige Zukunft. So kamen hierhin nach Gaeta wie später nach Rom Geistliche und Weltliche zu dem jungen Fürsten, um ihm ibre Wünsche auszusprechen oder Urkunden von ihm zu erlangen ${ }^{4}$ ), so dürfte sich auch unser Dichter nach dem von Eboli, bezw. Salerno nicht allzu fernen Gaeta begeben haben, um - wie einst Heinrich VI. ${ }^{5}$ ) in grösserem Stil - jetzt Friedrich II. seine poetische Huldigung darzubringen. Friedrich II. hatte bisher, seit ihn seine Mutter, die Kaiserin Constan $\iota$, spätestens Ende Dezember 11976) nach Messina hatte kommen

1) BF. 646 b. Dazu die scharfsinnigen Untersnehungen von H. Bloch, Die staufischen Kaiserwahlen und die Entstehung des Kurfürstentums. Leipzig-Berlin 1911, 89-108, auch für das Folgende.

2) BF. $650 \mathrm{a}$.

3) BF. 659 b. -- Hier in Gaeta stellt Friedrich als: „in imperatorem electus" für den Kardjnalpresbyter Petrus von S. Marcello betreffs einer Kapelle in Amalfi eine Urkunde ans (BF. 660. Gedruckt Winkelmann, Acta, I, p. 95 f., no 110); diescr 'litel zam ersten Mal im Februar 1212 in Messina: BF'.654.

4) In Gaeta erschienen ausser dem Kardinalpresbyter Petrus (vgl. Anm. 3) Graf Richard v. Fondi, die Herren von Aquino und Abgesandte der Leute von Rocea Bantre, die um Entsendung eines Kastellans baten (ef.: Rycc. de S. Germano, MG. SS. XIX, 334 f.); nach Rom kamen, un sich Schenkungen bestätigen zu lassen, der Abt von Casamari (BF. 665; gedr.: Winkelmann, Acta 1, p. 97, $\mathrm{n}^{0}$ 112) und der Abt von Casanova (BF 664, gedr.: Winkelmann, Acta I, p. $96 \mathrm{f}$, $n^{0} 111$; Casanova, ssw. von Penne, liegt etwa entsprechend weit von Rom, wie Eboli von Gaeta.).

5) Ausser dem ,Liber ad honorem Augusti « dürfte Peter wohl auch die ,Gesta Federici * Heinrich VI. gewidmet haben. Allerdings ist nichts UnbedingtSicheres über die Abfassungszeit dieses, Sanges von Friedrich Barbarossas Kreuzzug * zu sagen: jedenfalls ist er vor dem Gedicht „De Balneis entstanden. Da Peter aber zweifellos bei Heinrich VI. auf ein grösseres Interesse an der Verherrlichung seines Vaters als bei Friedrich II. rechnen konnte, hat man es mit vollem Recht für „wohl möglich erklärt, dass Heinrich VI.... den Dichter zu einem zweiten Gedicht auf seinen Heldenvater aufgefordert hat (Simonsfeld 71, Anm. 3 (72); rgl. auch Block II, 4. - Die Vollendung dieses Gedichts wäre dann wobl zu 1197 anzusetzen). - Beweise für diese Ansetzung liegen allerdings nicht vor.

b) Das hoffe ich bei andrer Gelegenheit nachweisen zu können; vgl. BF. 513, wo das Datum noch unentschieden gelassen wird. 
lassen, das sizilische Festland noch nicht betreten; so nrochten Peters Kenntnisse über des jungen Königs Leben nicht eben gründlich sein (eine Erklärung für die doch recht unpersönlich, allgemein gehaltene Widmung!); zudem drängte die Zeit, wenn er, ehe sich Friedrich nach Deutschland begab, noch dessen Aufmerksamkeit auf sich lenken und von ihm eine Belohnung erhalten wollte; was lag da näher, als das Gedicht "De Balneis", das fertig vorliegen mochte1), mit einer Widmung an Friedrich II. (in Anfangs- und Schlussstrophej zu verseben? (so erklärt es sich auch am einfachsten, dass sich sonst in dem Gedicht keinerlei Hinweise auf den hohen Adressaten finden, dass der behandelte, aus Peters medizinischen Interessen ${ }^{2}$ ) sich ergebende Stoff ja an sich nicht eben viel mit dem Staufer zu tun hatte). Dass wir von einer Belohnung durch Friedrich II. nichts wissen, worüber Block ${ }^{3}$ ) sein Befremden äusserte, ist an sich wenig auffällig, da eine derartige. Lücke in der Überlieferung ja nicht zu den Seltenheiten gehört - es

1) Die Widmungsstrophen (am Anfang und Ende des Gedichts) maclien mir durchaus den Eindruck einer späteren Hinzufügung, da sich sonst nirgends irgendwelche Andeutungen auf den Herrscher finden; vgl. dagegen die Anreden an die Kranken in den einzelnen Strophen mit , ros etc, z. B. Capaccio, l. c., I, p. 73, Z. 11 ; p. 77, Z. 21; p. 79, Z. 9; p. 82, Z. 7 etc. Besonders charakteristisch für diese Art der Entstehung des Gedichts scheint unir zu sein, dass Hinweise auf Friedrich II. im eigentlichen Text selbst da fehlen, wo sie sich fast von selbst ergeben, jedenfalls ganz leicht hätten einfügen lassen, 7. B. bei der Beschreibung des ,Balneum Imperatoris, quod Sol et Luna diciturs (Capaccio l. c. I, p. 79), die mit den Versen beginnt:

Caesaris est lavacrum quod Sol et Luna vocatur, sicut Sol stellis praevalet istud aquis",

um sich dann sogleich den Kranken, die hier Heilung finden, zuzuwenden. Wie leicht konnte an dieser Stelle eine Beziehung zwischen Friedrich II. u. den römischen Kaisern (denn nach diesen ist das Bad genannt; ef. Lombardo, Dovoụts eorum, quae de balneis aliisque miraculis Puteolanis scripta sunt... Venetiis MDLXVI, p. 59 (falsche Paginierung statt 63) und 64 und Percopo 599) hergestellt werden: - Dass das Stoffliche des Gedichts übrigens wenig Anspruch auf Originalität machen kann, hat P. Giacosa, Atti della R. Accademia delle scienze di Torino, vol. XLI (1906), p. 544 ff. dargelegt, der des Dichters direkte Abbängigkeit von einem Traktat des XII. Jahrhunderts : „Balnea Puteolana ‘, dessen Verfasser ein Arzt Johannes ist, nachgewiesen hat.

2) Peter hatte jedenfalls grosse medizinische Interessen und Kenntnisse; cf, Siragusa XVIf., Marletta, Arch. stor. per la Sicilia orientale, Anno VI (Catania 1909), 419; vielleicht war er doch sogar Arzt der Salernitaner Schule; cf. Rota XXI f. und Arch. Murat. Nr. 5, p. 277-280; Holder-Egger, N. A. XXXIV (1908), 244f., (cf. schon vorher: De Renzi 1, 287; Block I, 7-17; Percopo 617; Bigoni $10 \mathrm{f}$.) ; diese Annahme scheinen mir auch die Einwendungen Giacosas in seinen interessanten Ausfübrungen l. c., p. 542-554 nicht un beding t auszuschliessen.

3) Block I, 14 Anm. 1 (15); vgl. II, 2. 
scheint mir aber auch nicht einmal ausgeschlossen, dass Friedrich dieser Gelegenheitswidmung die gewünschte Belohnung versagte. Dass Peter von Eboli endlich (in V. 12) Friedrich II. verspricht, einst die Taten seines Sohnes zu besingen, würde sich dann vorzüglich daraus erklären, dass Heinrich (VII.) von seinem uach Deutschland ziehenden Vater als "gekrönter sizilischer König "1) zurückgelassen wurde, sein Leben also aller Voraussicht nach eher als das des Sizilien verlassenden Friedrich dem Dichter den Stoff zu einem neuem, vierten Poem hätte abgeben können.

Es bleibt mir nur noch übrig, darauf hinzuweisen, dass jedenfalls - auch wenn meine Datierungshypothese: Utherreichung des Gedichts "De Balneis" durch Peter von Ebboli an Friedrich II. im März oder April 1212 in Gaeta, auf irgendwelche Bedenken stossen sollte - die Vollendung dieses dritien Werks unseres Dichters erheblich ${ }^{2}$ ) vor die Kaiserkrönung Friedrichs fällt, dass mithin Friedrich II. auch nach der Anschauung unseres Dichters durch seine $\mathrm{Wahl}$ zum deutschen Kaiser, noch vor der päpstlichen Krönung, in die erlauchte Reihe der „Cäsaren" aufgenommen und auch dem "Namen" nach unter die Kaiser ${ }^{3}$ ) zu rechnen war - eine bei den gerade jetzt erörterten Fragen über die deutsche Kaiserwahl ${ }^{4}$ ) nicht uninteressante Feststellung. -

II.

Die folgende Untersuchung über die Lesarten der Schlussstrophe desGedichts "De Balneis“, speziell des Verses (3):

1) cf. BF 3835 c, 3836, 654 a. - Über die in dieser Tatsache liegende, Vorbereitung einer künftigen Lösung der für den Augenblick unvermeidlichen Personalunion $z$ wischen Kaiserreich und sizilisch'm Königreich vgl.: Winkelmann, Jahrbücher Philipps . . und Ottos IV . . , Bd. II, 316 f.; Bloch, Die stauf. Kaiserwahlen ...., 95; Hampe, Deutsche Kaisergeschichte im Zeitalter der Salier und Staufer (1909), 197.

2) Peters Tod spătestens Juni 1220. Vgl. S. 584. - Kaiserkrönung : 22. November 1220 ; BF. 1202 a.

3) Dass ,Cesar $\propto$ in unseren Versen nur $=$ „Imperator " sein kann, geht einmal aus dem Zeitgebrauch (vgl. Bloch, Die stauf. Kaiserwahlen 17 Anm. 4), ganz sicher aber aus der Gleichstellung Friedrichs II. mit Heinrich VI. (cf. oben) hervor. Anch ,Augustus" als selbständiger Titel kann doch nur als „Kaiser" gedeutet werden.

1) Vgl. Mario Krammer, Der Reichsgedanke des staufischen Kaiserhauses (Untersuchungen zur deutschen Staats- und Rechtsgeschichte, herausggb. von Gierke, 95. Heft, Breslau 1908), bes. 52-56; Bloch, Die staufischen Kaiserwahlen ..., bes. 89-108 (- Historische Vierteljahrsschrift XII (1909), bes. 516-535), Hampe, Kaisergeschichte, bes. 197. 
"Primus habet patrios civili marte triumphos" will das gedruckt vorliegende Material zu dieser Frage zusammenfassen, da die Textgestalt dieser Strophe, wie wir sahen, für unsere Untersuchung ron erheblicher Bedeutung war.

I. Es seien daher zunächst kurz die früheren Drucke der Schlussstrophe, soweit sie mir zugänglich waren, verzeichnet und charakterisiert:

1. a) Lombardo $=$ Eovoits eorum, quae de balneis aliisque miraculis Puteolanis scripta sunt auctore Joan. Francisco Lombardo Napolitano Venetiis MDLXVI (1566), $4^{0}$, p. $78 \mathrm{f}$. - Lombardo gibt den Text eines älteren Druckes (vgl. meine Anm. 1 S. 577) wieder (cf. p. 78), zieht aber ausserdem ein Manuskript des Klosters S. Severino zu Neapel zur Textherstellung heran (cf. seine Bemerkung l. c. p. 7); vgl. Huillard-Br. 335 - und bedient sich für die Verse $7 \mathrm{ff}$. eines Cod. Venet. (cf. seine Bemerkung p. 79), der aber nicht mit dem von Simonsfeld (cf. unten 4) benutzten identisch sein kann wegen starker textlicher Abweichungen.

b) (Elis.) $-\mathrm{Maz}_{.}=$Opusculum de balneis Puteolorum Baiarum et Pithecusarum a Joanne Elisio medico instauratum. Nunc denuo a Scipione Mazzella Napolitano recognitum pluribus rebus auctum et illustratum. Cum additamentis auctorum omnium qui hactenus de his scripserunt. Neapoli MDXCI (1591), $8^{0}$, p. 51. - Der Text in Mazzellas Anhang charakterisiert sich wohl als einfacher Abdruck der Ausgabe Lombardos, da genaue Übereinstimmung mit deren Wortlaut festzustellen ist; Mazzella selbst gibt, soviel ich sehe, über die Herkunft seines Textes keinerlei klare Auskunft (ef. aber seine Bemerkung p. 62). Vgl. auch Huillard-Br. 335 .

2. a) Cap. I = Balnearum quae Neapoli, Puteolis, Baiis, Pithecusis extant, virtutes. Thermarum et Balnearum apud antiquos structurae usus ministeria ... Neapoli MDCIV (1604) [Anhang zu Puteolana Historia a. Julio Caesare Capacio. Neapoli MDCIIII mit selbstilndiger Paginierung, $4^{0}$, p. 84. Dieser Druck beruht (ausser den früheren Drucken) auf einem alten Manuskript (p. 4: „manuscriptus codex, quem Joannes Simeon Moccia, totius antiquitatis arbiter, ex naufragio librorum Joannis Antonij Pisani celeberrimi nostra aetate medici servavit et quem hoc libro typis mandandum curavi ${ }^{\star}$ ) und hat selbständigen Wert.

b) Cap. II = La vera antichità di Pozzuolo descritta da Giulio Cesare Capaccio, Secretario dell' inclita città di Napoli, in Napoli MDCVII (1607), $8^{0}$, p. 347 f. - Wörtlieher Abdruck aus Cap. I.

3. Huillard-Br. 338 f. - aus selbständiger Handschrift (cf. Teil II (d) dieses Abschnittes).

4. Simonsfeld, 70 - aus selbständiger unvollständiger Überlieferung (cf. Teil II (e) dieses Abschnittes). 
Auf alle Varianten dieser früheren Drucke einzugehen, kann ich. mir ersparen, da sie z. T. von geringer Bedeutung sind. Nur die wichtigsten seieı hervorgehoben:

V. 1. Suscipe, Sol mundi tibi quem transmitto libellum: Lombardo = (Elis-) Maz. $\mathrm{Da}$ aber sämtliche uns bekannten übrigen Handschriften »presento a aufweisen (Cap. I $=$ Cap. II, Huillard-Br. (= Simonsfeld 72), Siragusa XIX), können wir diese Lesart als völlig gesichert verwerten (cf. meine Anm. 4 S. 584), zumal der Druck Lomb. auch sonst schlechte Varianten aufweist (vgl. V. $3,6,11)$.

V. 3. Vgl. Teil II dieses Abschnittes.

V. 6. tertius orbatas iste reformat aquas: Lomb. $=$ (Elis-) Maz. Der oben gegebene, handschriftlich (Huillard-Br. 338 Anm. 2, Siragusa XX) belegte Text gibt den richtigen Sinn.

V. 8, sei nebenbei gesagt, beruht auf Anlehnung an Deuteron. XVII, V. 6 ; XIX, V. 15; Matthäus XVIII, V. 16 u. a.

V. 10. Pauper in auster eo nemo poeta fuit: Simonsfeld $(\ldots$ austereo ... Huillard-Br. 338 Anm. 3) - sinnlos.

V. 11. Euboici vatis: Lomb. = (Elis-) Maz. Die andere Lesart (Cap. I-II; Huillard-Br., Simonsfeld, Siragusa XX; cf. auch Rota $\mathrm{XXV}$, Anm. 1) allein richtig.

II. Eine Zusammenstellung der Lesarten für V. 3 nach der handschriftlichen Überlieferung wird die Richtigkeit der im Abschnitt I unserer Untersuchungen verwerteten Fassung nachweisen:

a) Neapel, Bibl. Nazionale XIII, C, 37 - sec. XIV.

„Primus habet ptarios (patrios) inutilis arte triumphos

nach: Siragusa XXI, Anm. 2 (,ptarios“), Percopo 685 Anm. (,patrios“). Genaueres über die Handschrift: Percopo $621 \mathrm{ff}$.

b) Neapel, Bibl. Nazionale (wobl XIV, D, 18; cf. Percopo 611 Anm. 1

(p. 612) u. 623 Aum. 4) - sec. XV. (wenig später als a)

${ }_{\gg}$ Primus habet partes civilis in arte triumphi "

nach: Siragusa XXI Anm. 2; cf. Percopo 623 Anm. 4.

So auch in den Drucken von Lomb. 78 (der aber selbst schon im Kommentar (ebd.), jedoch scheinbar nicht auf Grund der ihm vorliegenden Lesarten, die Emendation „Primus habet patrios (!) sublimi (!) Marte triumphos" vorschlug) $=($ Elis-) Maz. 51; so auch in dem Druck Cap. I, $84=$ Cap. II, 347. Ob die von Lomb. oder von Cap. zur Textherstellung benutzte Handschrift (vgl. Teil I dieses Abschnittes) etwa mit b) identisch ist, vermag ich nicht zu entscheiden; einer von beiden 
Drucken beruht zum mindesten auf von b) unabhängiger Grundlage $\left(=b_{2}\right)$. Nach Bethmann, Archiv der Ges. f. ältere Deutsche Geschichtskunde, Bd. XII (1874), 524 sind die meisten Handschriften des Klosters S. Severino zu Neapel (eine solche benützte Lombardo) in die Bibl. Naz. übergegangen.

c) Rom, Bibl. Angelica, antica V, 2, 11; moderna 1474 - sec. XIV. "Primus habet patrios civili marte triumphos"

nach: Siragusa XXI Anm. 2; vgl. dazu Paciaudi, op. cit., 51 (der wohl diesen und einen ebeudort nicht mehr vorbandenen Codex durcheinanderwirft; cf. Percopo 621 Anm. 1; ef. auch Bethmann, l. c. 377, nicht 379, wie Percopo 1. c. augibt).

d) Paris, Bibl. Nation. Cod. 7471. Anciens fonds franç. (fonds du roi) - sec. XIV.

„Primus habet patrios civili marte triumphos"

nach : Huillard-Br. 338 u. Anm. 1; cf. über die Handschrift: HuillardBr. 344 u. 348 und Percopo 620 Anm. 4.

e) Venedig, Marc. XI, 124 - sec. XVI. (1503; Copie nach dem Chronicon Marci, in das unser Gedicht aufgenommen wurde und das Ende des XIII. Jahrhunderts verfasst wurde; ef. Simonsfeld 53, 73, auch schon Bethmann-Waitz, N. A. II, 350, 351, 354)

$$
\text { "Primus habet patrios civili in arte triumphos" }
$$

nach: Bethmann-Waitz, 354; Simonsfeld 70.

f) Cod. Estensis (etwa = Modena, Bibl. Estensis V, C, 2\%? cf. Bethmann, Archiv XII, 697: allerdings verzeichnet er im Gegensatz zu Tiraboschis Angaben (l. c. 352): "Alcadinus" de baln. Puteol. (Alcadinus wurde früher das Gedicht zugeschrieben); diese Überschrift etwa neueren Datums?)

„Primus habet patrios civili Marte triumphos "

nach: G. Tiraboschi, Storia della letteratura Italiana, Tomo quarto (Modena 1774), Libro III, 352. - Die Bethmann, Archiv XII, 415 namhaft gemachte Handschrift (Handschriften des Can. Rossi in Rom), mbr. oct. sec. XIII ex. ist nach Percopo $621 \mathrm{Anm} .1$ verschollen.

In dem Cod. Pavia, Bibl. Universitaria $\mathrm{n}^{0} 488$, f. 61, der unser Gedicht enthält, fehlt das letzte Epigramm: unsere Dedikationsstrophe; cf. Rota XXVI, Anm. 1.

Ausserdem macht noch Paciaudi, op. cit, 51 auf 3 vatikanische Codices aufmerksam: $\alpha$ ) Vatican. Ottobon. - (ohne nähere Angabe) 
- $\beta$ ) Cod. Vat. 1528 membr. saec. XIV - $-\gamma$ ) Cod. Vat. 3436 - cartac. saec. XV - und ebd. 50 auf einen Cod. membr. saec. XIII ex. in Neapel, in pluteis bibliothecae nostrae domus S. Paulli". Näheres teilt er nicht mit (zumal er sich mit der uns interessierenden Strophe garnicht beschäftigt). - Ich notierte diese Handschriften nur, um möglichste Vollständigkeit im gedruckten Material (solehes allein konnte ich berücksichtigen) zu erreichen. - Ich möchte noch hinzufügen, dass $\alpha$ ) viell. = Cod. Vat. Ottobon. $2110 \mathrm{mb}$. (De balneis Puteoli, Bethnann, Archiv XII, 368) sein könnte, dass endlich (cf. Bethmann ebd. 523), ein Teil des Handschriftenbestandes von S. Paolo in Neapel in die dortige Bibl. Nazionale übergegangen ist.

Ich fasse zusammen: die Fassung "patrios civili marte triumphos" ist durch die Handschriften c), d), e), f) belegt, durch a) nahegelegt (bezw. auch bewiesen); nur Handschrift b) und die älteren Drucke weisen "partes", überhaupt keine Handschrift und ke in älterer Druek "partos" auf 1$)$. -

\section{III.}

Friedrichs II. Urkunde für das Erzbistum Salerno bildete eine wichtige Grundlage für die Untersuchungen des I. Abschnittes ${ }^{2}$ ) und bedarf daher noch einer kurzen gesonderten Betrachtung.

Die Urkunde ist gedruckt: Huillard-Bréholles, Historia diplomatica Friderici secundi, Tom. II, pars I (Paris 1852), $111 \mathrm{ff}$. (nach Register im erzbischöđlichen Archiv zu Salerno) und G. Paesano, Memorie per servire alla storia della chiesa Salernitana, Parte seconda, Salerno 1852, $316 \mathrm{ff}$. (nach Bestätigung von 1287, über deren Original vgl. Siragusa IX, Anm. 1). Die uns interessierende Stelle lautet (Huillard-Br. 113, Paesano 318, Siragusa IX f.): [Friedrich bestätigt der erzbischöflichen Kirche] .... molendinum de Albiscenda (Huillard-Br. irrtümlich Abescenda) in Ebulo (Paesano: Ebolo) consistens, quod magister Petrus versificator a clare memorie domino Henrico imperatore Romanorum patre nostro iure hereditario habuit, tenuit et in fine vite sue idem magister Petrus illud sancte Salernitane ecclesie donavit pariter et legavit.... - Siragusa IX und Marletta 111 setzen die Urkunde in den Februar 1220, Rota XX (und Anm. 1) und danach Chalandon

1) Die ron Marletta 107 Anm. 3 versprochene Neuausgabe des Gedichts ,De Balneis ", die auch über diese Frage endgültig Klarheit schaffen dürfte, ist meines Wissens noch nicht erschienen, war mir jedenfalls auf deutschen Bibliotheken nicht zugänglich.

2) Vgl. Seite 581, 584 . 
(Histoire de la domination Normande en Italie et en Sicile, Tome premier (Paris 1907), Introd. p. LXI) ebenfalls zu 1220, und zwar alle auf Grund des (mir nicht zugänglichen) Schriftchens: „Augelluzzi, Intorno ad alcuni maestri della scuola Salernitana, Napoli 1853, p. 6, nota $2^{4}$ (Augelluzzis Behauptung fusst anf Einsicht in das Original der Bestätigung von 1287 (cf. oben)). Trotzdem ist die Datierung 1221 (BF. 1280) festzuhalten (cf, auch Percopo 615 und Anm. 3). Allerdings heisst es in der Urkunde: anno dominice incarnationis MCCXX (so auch: Huillard-Br.115; Paesano, 1.c. 320) und indictioneVIII (so Siragusa IX; dagegen Huillard-Br. 115 und Paesano 320: indictione IX, resp. nona), aber die übrigen Zeit- und Ortsangaben: Datum Capue post curiam celebratam.... imperii domini nostri Friderici Dei gratia Romanorum imperatoris semper augusti anno primo .... (Huillard-Br. 115, Paesano 320) und die Tatsache, dass „Fridericus divina favente clementia Romanorum imperator" die Urkunde ausstellt (Huillard-Br. 111) und ,domina Constantia coniux nostra Romanorum imperatrix* (Huillard-Br. 112; Marletta 111) erwähnt wird, geben den Ausschlag für Februar 1221 (Kaiserkrönung: 1220 Nov. 22; BF 1202a).

Es mögen im Anschluss an diese Urkunde noch einige Bemerkungen folgen: Die hier erwähnte erbliche Verleihung der Mühle (iure hereditario) durch Heinrich VI. an Peter hat noch den Grund zu Streitigkeiten gegeben. Peter von Eboli, selbst als Geistlicher ohne Nachkommenschaft, hat, wie wir sahen, in seinem Testament die Mühle nicht seinen Verwandten, sondern der erzbischöflichen Kirche von Salerno vermacht. Aber dieser wurde der Besitz später durch die Söhne des Richters Petrus de Ebulo, zweifellos eines Verwandten unseres Dichters Peter, strittig gemacht, dann jedoch durch Urteilsspruch des kaiserlichen Grosshofgerichts von 1245 Januar (Foggia) auf Grund genauer Untersuchung wieder zugesprochen, indem die Brüder zur Herausgabe der Mühle (... molendinum, quod est situm in terra Ebuli in loco, ubi Albiscenda dicitur ...) gezwungen wurden 1 ).

Dass deren Vater, der Richter Petrus de Ebulo (,iudex"), etwa mit unserem Dichter identisch sein köune, bestreitet Siragusa XV mit Recht; im Bulletino dell' Istituto Storico Italiano, Nr. 30 (Roma 1909), p. $41-46$ führt er noch einmal den ausführlichen Beweis für diese Tatsache.

t) Der Urteilsspruch ist doch wohl zu 1245 Januar anzusetzen (vgl. BF. 3454), nicht 1244, wie Siragusa (XV und, Giunte, chiarimenti e correzioni all' edizi one del , Liber ad honorem Augusti di Pietro da Eboli «, Bulletino dell' Istıtuto Storico Italiano, Nr. 30, Roma 1909, p. 43) und Rota XXIII angeben. Gedruckt: Paesano, op. cit., II, 353; Huillard-Br., Hist. dipl. Frid. sec., Tom. VI, pars I, :-50 über das Original cf. Siragusa, ,Giunte ...*, 43. 


\section{P.P.}

Im Verlage der Wagner'schen Universităts-Buchhandlung in Innsbruck ist soeben eine historische Darstellung zur Ausgabe gelangt, die mit Hülfe eines reichen, vielfach neuen und unbekannten Quellenmaterials aus tirolischen, süddeutschen, schweizerischen und italienischen Archiven

$$
\text { „Beiträge zur Geschichte }
$$

der Lostrennung der Schweiz vom Deutschen Reiche"

von neuen Gesichtspunkten aus liefert.

Das umfangreiche Werk ist betitelt:

\section{Die geächteten Räte \\ des \\ Erzherzogs Sigmund von Österreich \\ und \\ ihre Beziehungen zur Schwriz \\ $1487-1499$ \\ von \\ Dr. phil. Friedrich Hegi \\ II. Stoutsarchivar zu Zarich}

und kostet K. 25.- oder M. 22.-

Der 1. Teil schildert die Mißwirtschaft der vielköpfigen und aus den verschiedensten sozialen Elementen zusammengesetzten Günstlingsclique am Hofe des schwachen Erzherzogs Sigmund v. Österreich zu Innsbruck in den Jahren 1478-1487, die beinahe zur Einverleibung Tirols und Vorderösterreichs in die Gebiete der Herzöge von Bayern gefuhrt hätte, stellt die Herkunft und Schicksale der bisher noch 
nicht näher bekannten „ungetreuen" Räte fest und untersucht mit positiven Ergebnissen deren Majestätsverbrechen, Hoch- und Landesverrat.

Die Namenliste der geächteten Räte mag beweisen, daß das Werk für Österreich, Deutschland, die Schweiz und Oberitalien vorzüglich in Betracht kommt. Es sind:

Georg, Graf v. Werdenberg und Sargans, Pfleger zu Landeck; --- der Obersihofmeister und Feldhauptmann im Kriege gegen Venedig, Vogt Gaudenz v. Matseh (Amatia, conte di Colonna), Graf zu Kirchberg; - Oswald, Graf zu Tierstein, vorderösterreichischer Hauptmann und Landvogt und $\mathrm{Er}$ b a u er d er Ho h k ö n i g sburg; - Heinrich, Graf zu Fürstenberg der Jüngere; -- Hans Werner, Freiherr v. Ziminern, Hauptmann der Herrschaft Hohenberg: -- der Ritter Hans v. Wä. hingen, Bürger in Rottwei], Obermarschall und Vogt der obern Herrschaft Hohenberg; - der Kämmerer und Sekretär Ulrich v. Göggingen aus Überlingen; Jakob Streit aus Villingen, Vogt zu Triberg; - Paul Marquart aus Stockach; der Kanzler und Dekan zu Kaltern, Hans Schweikle genannt v. Jenalz aus dem Sarganserlande; - der Sekretăr Dr. jur. Christian Winkler; - die Hofmeisterin Anna Spieß v. Friedberg; - der Kammerknecht Gotthart Hartlieb v. Stauheim (Bayern), Pfleger zu Fragenstein; - der bayerische Kämmerer Thoman Pipperle; - der Hofmarschall, Hauptmann auf Nons und Sulz, Hildebrand v. Cles; - der Vogt zu Bregenz, Lippman v. Meusbach aus Leipzig.

Die folgenden Teile befassen sich, immer unter Berücksichtigung der auswärtigen Verhältnisse, mit der Intervention der schweizerischen Eidgenossenschaft für die seit 1488 in die Reichsacht erklärten obigen erzherzoglichen Räte. Sie legen dar, wie dieses merkwürdige Eintreten von Gliedern des Deutschen Reiches gegen Kaiser und Reich wesentlich mitgeholfen hat zur Entfremdung der Schweiz vom deutschen Reiche, zur Festigung der schweizerischen Souveränität und zum Ausbruch des sog. Schwabenkriegs. Ein gewisses aktuelles Interesse bieten gerade diese Feststellungen angesichts von wirklich vorhandenen, wenn auch nur schwach sich bemerkbar machenden Trennungstendenzen unter den drei Völkerstämmen der heutigen Schweiz. - Für die Geschichte des schweizerischen Asylrechtes, für das Söldner-und Pensionenwesen sind ebenfalls neue Ergebnisse gewonnen.

Für die habsburgische Monarchie sind die Beleuchtung des Übergangs Tirols und Vorderösterreichs an König Maximilian 1490, für Bayern die des Neutralitătsbündnisses von 1491 mit den Eidgenossen, für Italien der Krieg gegen Venedig 1487 und die Beziehungen einzelner Geächteter zu den Herzögen von Mailand, für 
die Kirchengeschichte die Verwendung des päpstlichen Stuhles für die Geächteten von Interesse.

Rechts- und Wirtschaftsgeschichte wie allgemeine Kulturgeschichte ziehen aus diesem Zeitgemälde, dem verzweiflungsvollen Ringen untergehender Dynastengeschlechter inmitten neuer politischer, wirtschaftlicher und sozialer Formen erheblichen Gewinn.

Zwei Illustrationstafeln sind dem Werke beigegeben, die eine stellt nach einem zeitgenössischen Bilde die Flucht der gestürzten Räte aus der Stadt Hall, die andere ihre Siegel dar; eine V erw andt. schaftstafel orientiert über den verwandtschaftlichen Zusammenhang einzelner der Geächteten.

Ein ausführliches Personen-, Orts- und Sachregister schließt den Band.

Bemerkt sei noch, daß Se. Majestät der Deutsche Kaiser als Eigentümer der Hohkönigsburg huldvoll geruht hat, ein Exemplar des Werkes aus der Hand des Verfassers entgegenzunehmen.

Hochachtungsvoll und ergebenst

Wagner'sche Universitäts-Buchhandlang in Innsbruck.

\section{Bestellschein.}

Der Unterzeichnete bestellt hiedurch bei der Buchhandlung

\section{Hegi, Die geächteten Räte des Erzherzogs Sigmund von D̈sterreich u.s.w.}

Preis K. 25.- oder M. 22.-

Adresse:

Name: 
Brought to you by | Nanyang Technological University Authenticated

Download Date | 6/11/15 1:17 PM 
Ich möchte an dieser Stelle betoneu, dass ich den Feststellungen Siragusas über Peters Persönlichkeit im Wesentlichen durchaus beistimmen zu müssen glaube. Die Identifizierung unseres Dichters mit Petrus Ansolinus (in Urkunde Friedrichs 1I. für das Kloster Montevergine, 1219 Mai)1), wie sie Rota in seiner Ausgabe (sogar im Titel, den er dem Gedicht gibt (vgl. meine Anm. 2 S. 576) u. p. XXf.) vertritt und in seiner Besprechung von Siragusas Ausgabe ${ }^{2}$ ) zu verteidigen sucht, ist doch sehr gewagt, zum mindesten in keiner Weise bewiesen oder bei dem vorliegenden Material beweisbar. Siragusa, Marletta (der die von Rota angeführten Beweisgründe für diese Annahme einzeln zurückweist) und Holder-Egger ${ }^{3}$ ) bestreiten die Identität, wie mir scheint, mit Recht. Selbst wenn Beinamen wie Ansolinus in dieser Periode schon vorgekommen $\operatorname{sind}^{4}$ ), ist doch noch keineswegs sicher, dass gerade unser Peter (BF. 2542 erwähnt z. B. einen sicher nicht mit unserem Dichter identischen Magister Petrus de Ebulo) diesen Beinamen geführt hat, und auffällig bleibt das Fehlen des "Ansolinus" in Peters eigener Unterschrift im "Liber ad honorem Augusti“ doch auf alle Fälle; dieses Bedenken aber würde auch bestehen bleiben, wenn wir (was an sich richtiger sein dürfte) die Form "Ansolini“ (vgl. Anm. 1) als „Sohn des Ansolinus" fassen würden (vgl, auch Siragusa Xf.). Die Gleichung: "magister Petrus (versificator) de Ebulo" $=$ "magister Petrus Ansolinus (resp. Ansolini) de Ebulo" erscheint mir, wenn nicht durchaus unmöglich, so doch unwahrscheinlich und unbeweisbar. Unbedingt sicher kann man demnach, wie gesagt, unseren Dichter (vgl. seine Unterschrift: Ausgabe Siragusas, p. 116) nur mit dem ,magister Petrus versificator" der mehrfach genannten Urkunde Friedrichs vom Februar 1221 identifizieren.

1) BF. 1016 (Augsburg): Friedrich bestätigt u. a.: , magistri Petri Ansolini de Ebulo unam vineam cum terra vacua in pertinentiis ipsius Petri ac septem petiolas terrarum in pertinentiis eiusdem ... Huillard-Br., Hist. dipl. Frid. sec., tom. I, pars II, 632; Siragusa X.

2) Archivio Muratoriano, Nr. 5 (1908), 276 f. - vgl. schon vor Rota dieselbe Ansicht bei Winkelmann, Des Magisters Petrus de Ebulo Liber ad honorem Augusti, Leipzig 1874, Einleitung, p. $11 \mathrm{f}$. und ihm folgend: Carini, Arch. stor. Siciliano, N. S. I, 490.

s) Siragusa $X$ ff., bes. XII; Marletta 110-117; Holder-Egger, N. A. XXXIV (1908), 244 f.; cfr. auch Chalandon, l. c., Introd. p. LXII.

4) Die von Rota, Arch. Mur., Nr. 5 (1908), 276 und Anm. 2 dafür zum Beweis herangezogene Vergleichung mit den Beinamen der deutschen Heerführer und der königlichen Familie ist nicht eben schlagend. 